\title{
Psicología de la corrupción: Balance de estudios realizados en el Perú, alternativas metodológicas de investigación
}

\author{
Fabio Anselmo Sánchez Fores. ${ }^{2}$ \\ Universidad Andina del Cusco, Perú \\ «Quien critica un problema sin proponer una solución, \\ es parte del problema que critica.» \\ Facundo Cabral \\ (1937 - 2011)
}

\section{RESUMEN}

En el presente artículo se analizan los principales estudios psicológicos realizados en el Perú sobre la corrupción: sus bases teóricas, procedimientos y resultados; el fin es ponderar la confiabilidad y objetividad de los mismos, así como, la validez y trascendencia de sus conclusiones. Se estudian sus causas y consecuencias desde otras disciplinas científicas conexas. Asimismo, se proponen alternativas metodológicas para la investigación científica — siguiendo el método hipotético-deductivo- de la conducta corrupta y sus factores psicológicos implícitos con el objetivo de sentar las bases para la explicación psicológica de su génesis, proceso y desarrollo — todavía inviables_-; de manera que se dispongan de las condiciones teóricas suficientes para predecir la probabilidad de su ocurrencia - personal y, per se (por sí mismo), institucional-, y así poder evitar sus consecuencias tan nocivas para el desarrollo de la sociedad y del país.

Palabras clave: corrupción, psicología de la corrupción, método hipotético-deductivo, epistemología de la psicología, método científico.

\footnotetext{
${ }^{2}$ Psicólogo, Licenciado en Filosofía y Maestro en Educación Superior. Docente de la Escuela Profesional de Psicología, Universidad Andina del Cusco, Perú.
} 


\begin{abstract}
Pychology of corruption: Balance of studies in Peru, methodologycal alternatives in reserch.

In this article the main psychological studies conducted in Peru on corruption are analyzed: his theoretical bases, procedures and results; to weigh the reliability and objectivity of the same, and the validity and also significance of their findings. Its causes and consequences are studied from other related scientific disciplines. Also, proposes alternative methodologies for scientific research -following the hypothetical deductive method - of the corrupt behavior and its underlying psychological factors, to lay the foundations for the psychological explanation of its genesis and development process, —-still viable-; in this way to provide the sufficient theoretical conditions to predict the probability of their occurrence personal, per se (for himself), institutional-, and so as to avoid harmful consequences for the development of society and the country.
\end{abstract}

Key words: corruption, corruption psychology, hypothetical-deductive, epistemology of psychology, science method.

\title{
INTRODUCCIÓN
}

Antes de iniciar el análisis, es preciso señalar algunos contrastes de lo que suele entenderse por corrupción (el mismo que puede ser concebido en una variedad de usos para denotar la conducta humana, desde la esfera privada hasta la pública) en sus distintas variantes, a fin de extraer la esfera en la que se enfocará el presente estudio, pues al tratarse de un fenómeno bastante complejo (por sus formas, alcances y perspectivas) la precisión no solo se hace importante, sino imprescindible.

Es así que el presente ensayo se orienta, inicialmente, al análisis general de la corrupción en la esfera pública, pues el fenómeno trae consecuencias nocivas para el desarrollo del país en su variante social y económica, siendo el principal obstáculo para su desarrollo. 
En tal sentido, se soslayará por el momento, su análisis en la esfera privada, pues ello amerita otro espacio de indagación, ya que implica variantes y variables que - no necesariamente - se hallan incluidas dentro de su ocurrencia en la esfera pública, pues, verbigracia, un funcionario que hurta millones de soles al erario nacional puede desempeñarse como un buen padre o un buen esposo. Para muestra, vale retrotraer a la memoria a los funcionarios nazis que se hallaban encargados de los hornos crematorios para los judíos en el holocausto: tales individuos realizaban actos inhumanos y de extrema crueldad en la esfera pública, pero, a su vez, se caracterizaban por ser excelentes padres y desempeñar sus funciones paternas y familiares sin la menor muestra de culpa y sin faltar a sus roles en tanto miembros y referentes principales de sus hogares.

Luego de la definición inicial de la corrupción, se pretende analizar los estudios psicológicos realizados hasta la fecha en nuestro país. Respecto de su metodología, sustento teórico y basamento epistémico, para conocer la objetividad y alcances científicos de sus conclusiones. Se hace esto con la finalidad de dilucidar el valor de tales estudios, a fin de proponer —en caso de que sean defectuosos - alternativas metodológicas en el estudio psicológico de este problema social, asumiendo que la psicología tiene la capacidad de explicar la conducta humana en todas sus variantes, no debiendo ser la conducta corrupta una excepción, y más aún, tratándose de un problema que agobia a nuestro país desde mucho antes del inicio de la era republicana hasta nuestros días, donde el problema parece haberse agudizado, extendiendo las redes de corrupción desde los estratos políticos más altos e influyentes de gobierno, hasta los menos determinantes.

\section{LA CORRUPCIÓN EN LA ESFERA PÚBLICA}

Es así que en el presente análisis se realizará un estudio sobre el fenómeno de la corrupción en la esfera pública de las organizaciones, el mismo que es definido por el Diccionario de la Real Academia de la Lengua Española (DRAE, 2014), como la 
«práctica consistente en la utilización de las funciones y medios de aquellas en provecho, económico o de otra índole, de sus gestores.» (DRAE, 2014).

Asimismo, según Bayley (1966), Mc Mullan (1961) Nye (1967), y Tilman (1968), (citados en: Gonzales, 1985), una conducta corrupta en la esfera pública se da cuando:

a. Actúa violando su responsabilidad hacia, por lo menos, un sistema de orden público o cívico, y es de hecho, incompatible o destructivo de tales sistemas.

b. Hace uso indebido de su autoridad.

c. Acepta dinero por hacer lo que tiene la obligación de hacer, o por no hacer lo que tiene la obligación de hacer.

d. Favorece a quien lo soborna.

e. Permite que en su toma de decisiones primen consideraciones de ganancia personal, de status o prestigio para su familia, grupo o partido.

\section{LA CORRUPCIÓN EN EL ESPACIO INTERNACIONAL, NACIONAL Y LOCAL}

Según datos de diversas entidades nacionales e internacionales (Transparency International, 2013; United Nations Office on Drugs and Crime - UNODC, 2015), los índices de corrupción a nivel global tienden a incrementarse cada año. La utilización o malversación de los fondos en beneficio propio (personal o grupal) son la práctica más frecuente.

Tales actos devienen en más perjudiciales cuando se realizan a nivel de las organizaciones públicas, pues las mismas tienen la finalidad de promover y propiciar el desarrollo del país. Es por ello que, cuando el dinero de las arcas del estado o del tesoro nacional en lugar de utilizarse para el beneficio de millones de personas se utilizan para el beneficio personal o los intereses particulares de unas cuantas autoridades, que aprovechando su situación de poder y de las deficiencias en los 
organismos de control del estado — cuando no de complicidad—, privan a un número considerable de ciudadanos de acceder a los beneficios que se podrían estar haciendo un uso correcto y justo de tales fondos económicos; esto además de desacelerar el crecimiento económico, genera inestabilidad política y debilita las instituciones democráticas. Se crea, en suma, perjuicios irreparables y paraliza el progreso de la nación (UNODC, 2015).

Es de lamentar que hechos como el descrito tienen una ascendencia arcana en la historia de nuestro país. Sus registros se remontan a la época colonial y republicana (que es a partir de la cual se puede hablar, en sentido pleno, de una conciencia de nación), que es en la que nos encontramos inmersos. Vale recordar los actos más conocidos, tales como la polémica huida del presidente del Perú durante la Guerra del Pacífico (1879 - 1883), Mariano Ignacio Prado (1839 - 1934), quien, so pretexto de comprar navíos de guerra en Europa, que abastecieran al alicaído ejército nacional (sobre todo después del hundimiento del Huáscar y el deceso heroico de su capitán, Miguel Grau Seminario, el Caballero de los Mares), permaneció en Estados Unidos (mas no en Europa, como estaba previsto) con todo el dinero encomendado, habiendo con ello, no solo engañado, sino estafado a toda una nación de la que fuera autoridad suprema y en quien se tenía depositada la esperanza de quizá virar el curso de la guerra en beneficio de todo un país, y no terminar con la derrota de la guerra y la pérdida de su dignidad y territorio (Tacna y Arica) tras la firma del Tratado de Ancón (a todas luces humillante y vejatorio para la dignidad nacional) (Basadre, 2005).

Aun habiendo pasado más de cien años desde entonces, tales hechos resultan familiares en la actualidad, verbigracia: la renuncia de Alberto Fujimori (ex presidente del Perú en los periodos, 1990 - 1995 y 1995 - 2000), vía fax desde el Japón, luego de su polémica huida para evitar su detención por los severos actos de corrupción durante su gobierno, en complicidad con su asesor y amigo íntimo Vladimiro Montesinos. 
Tales sucesos parecieran limitarse al espacio nacional y solo atañer a las autoridades presidenciales; sin embargo, el caso de Gregorio Álvarez (ex presidente regional de Ancash), encerrado por actos de corrupción en la Región Ancash, el de Hugo Gonzales Sayán (ex presidente regional de Cusco), condenado a ochos años de prisión por hechos tanto o más graves que el de aquel, etc., entre otras numerosas autoridades nacionales (hace un año el $92 \%$ de los alcaldes del país, casi 1700 de 1 841, estaban siendo investigados por presuntos actos de corrupción vinculados a los delitos de peculado de uso, malversación de fondos, negociación incompatible y colusión), condenadas por actos de corrupción; nos indican que los mismos no solo se hallan presentes en las esferas más altas del gobierno, sino en el espacio nacional en general, generando cuantiosas pérdidas económicas (10 000 millones al año, que representa el $2 \%$ del PBI nacional), anquilosando el desarrollo a un país (Contraloría General de la República, 2014), que por sus propias características necesita del trabajo honesto de sus autoridades en bien de los gobernados, dado que hurtar o estafar al país equivale a hacerlo a los pobres haciéndolos más pobres, en beneficio de unos cuantos, que muchas veces no alcanzan condena ni punición alguna, sumiendo en la crisis a nuestro país y agravando con ello su situación de pobreza y retraso social y económico. 
Fig. 1. Percepción de la corrupción entre funcionarios públicos (Macera, 2015).

Como se puede observar en el siguiente gráfico, el Perú ocupa el quinto puesto a nivel de Latinoamérica, en percepción de la corrupción, por encima de la mayoría de los países de la región. Un hecho a todas luces preocupante, en vista de que este resultado crea un clima de desconfianza, estrés e inseguridad en los peruanos, predisponiendo al brote de cuadros psicopatológicos, como trastornos del estado de ánimo (ansiedad, depresión), entre otras de sus variantes relacionadas con el afecto.

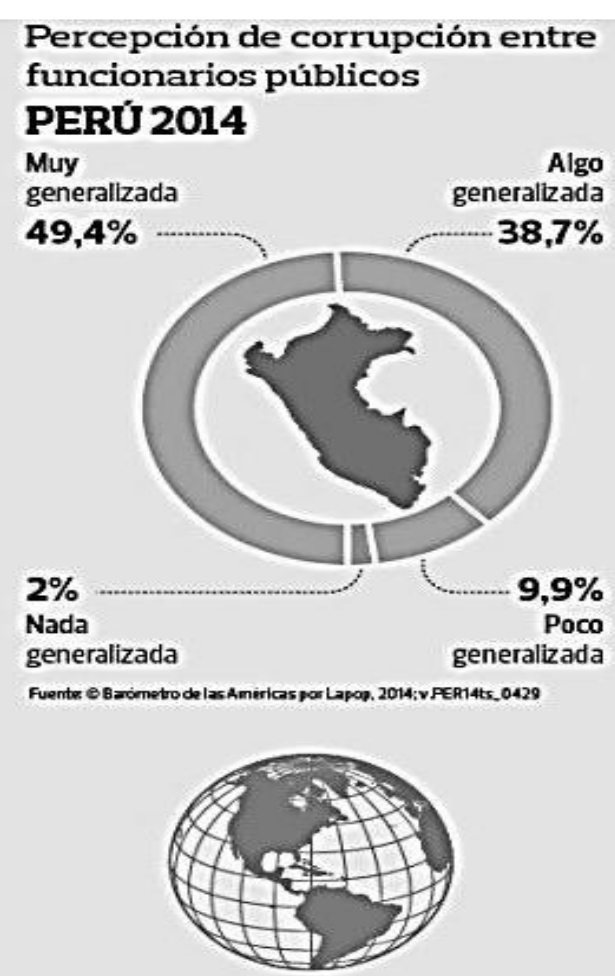

Percepción de corrupción en los paises de la región

2014

Venezuela $80 \%$

Colombia $79,6 \%$

Argentina 79\%

Guryana $\quad 78,4 \%$

Perú $78,2 \%$

Jamaica 78, $7 \%$

Paraguay $77,8 \%$

R. Dominicana $76,3 \%$

México $75,1 \%$

Belice $74,6 \%$

Guatemala 70,2\%

Panamá $69,4 \%$

Bolivia $68 \%$

ElSalvador 67,7\%

Honduras $67,3 \%$

Estados Unidos 66,3\%

Nicaragua $65,9 \%$

Ecuador 64,1\%

Uruguay $62,4 \%$

Haiti $62,7 \%$

Canadá $61,8 \%$

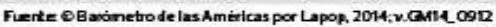




\section{CAUSAS DE LA CORRUPCIÓN}

Para el historiador Álvaro Gonzales Riesle (1985), las causas de la corrupción (se señala lo más relevante) son:

a. El subdesarrollo.

b. La situación general de anomia que conduce a la gente a pensar que cualquiera que ocupa una posición de poder va, muy probable, a explotarla en el propio interés, en el de su familia u otros grupos sociales hacia los que tiene un sentimiento de lealtad.

c. La existencia de una profunda brecha entre el gobierno y la sociedad.

d. La ausencia de un proyecto nacional y de lealtad del ciudadano hacia una sociedad organizada.

e. La expansión de la autoridad gubernamental y la multiplicación de actividades sujetas a regulación del gobierno.

f. Las acciones de empresas transnacionales que buscan decisiones gubernamentales que las favorezcan.

g. Un bajo nivel de disciplina social.

h. Una debilidad de los mecanismos de reforzamiento social y gubernamental.

i. La prevalencia de desigualdades en la distribución de la riqueza y de una considerable pobreza absoluta.

j. La nula diferencia entre el rol público y el interés privado aunado a una tendencia a ver la función pública como el medio principal de enriquecerse.

k. En suma, a mayor caos: subdesarrollo, desorientación, desintegración, dependencia del exterior, indisciplina, desigualdad, ignorancia y deshonestidad, mayor será también la corrupción.

Es importante destacar que según este análisis sobre las causas de la corrupción, se presentan:

- Primero, aspectos más relacionados con las consecuencias de la corrupción, tal y como la prevalencia de las desigualdades a las que predispone cayendo en un error de criterio, pues se toma la consecuencia por la causa. 
- Segundo, en ninguno de los puntos mencionados se hace alusión alguna a causas de orden psicológico, pues se consideran más factores de orden social, político y económico; pero ninguna que contenga atisbos de índole psicológica, como las patologías que podrían estar asociadas, verbigracia: la sociopatía.

Esta omisión responde a una carencia de estudios dentro de la psicología, lo que predispone a vacíos teóricos dentro de esta ciencia, de ahí que se omitan los aportes que se podrían hacer desde ella al esclarecimiento y explicación de la conducta corrupta, un hecho sobre el que se abundará más adelante.

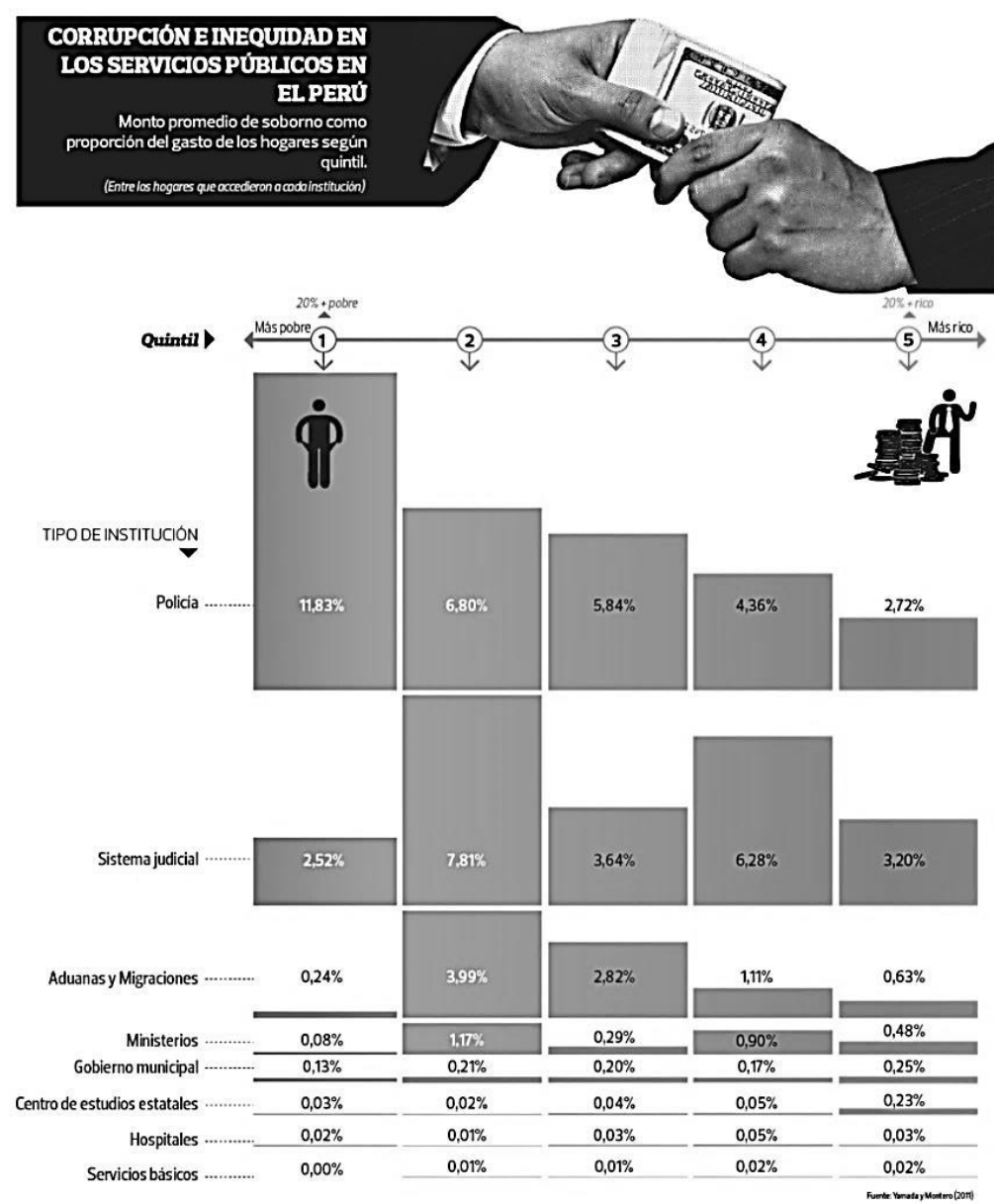

Fig. 2. Corrupción e inequidad en los servicios públicos en el Perú (Macera, 2015).

Como se puede observar en el siguiente gráfico, los mayores índices de corrupción en el Perú, se registran en la policía, el sistema judicial, en aduanas y migraciones, los ministerios, gobiernos regionales, centros de estudios estatales, hospitales y servicios básicos. Un hecho que refuerza las aseveraciones que sitúan la mayor incidencia de corrupción en las principales instancias de poder del Estado, que en lugar de generar orden y transparencia en sus procedimientos y procesos administrativos, obnubilan y corroen la confianza, el clima social y, per se, la salud emocional de los ciudadanos. 


\section{CONSECUENCIAS DE LA CORRUPCIÓN}

Para Gonzales (1985), tres son las principales consecuencias que la corrupción trae consigo, tales son:

1. En el orden político:

Genera una situación general de injusticia, ya sea por la escasa aplicación de la ley o a causa de medidas represivas. Hechos que reducen el respeto, la confianza y la legitimación del gobierno, a lo que se agrega la reducción de la estabilidad política, propiciando los golpes de estado haciendo, en suma, insostenible la continuidad de la democracia.

2. En el orden económico:

Ocasiona fuga de capitales nacionales, propicia la pérdida neta para los países en desarrollo y origina la privación de ayuda extranjera debido al repudio de los donantes hacia la corrupción en un país en desarrollo. Asimismo, distorsiona y desalienta las inversiones, en especial la extranjera, porque la corrupción constituye un riesgo debido a que el costo no oficial para comenzar una empresa es demasiado alto para ser rentable.

3. En el orden administrativo:

Promueve una situación general de ineficiencia administrativa (los contratos se adjudican a contratistas equivocados, las investigaciones no producen descubrimientos, el trabajo en grupo y la lealtad se desintegran). Asimismo, se desvían los recursos de propósitos públicos a propósitos privados de políticos y funcionarios, se incrementan los sobornos, se debilita la incorruptibilidad de un funcionario en una situación de corrupción generalizada, lo cual incrementa el problema y lo hace crónico. 
Por lo tanto, las consecuencias suelen ser nefastas para la sociedad en la que se instauran las redes de corrupción, lo que genera una situación altamente perniciosa, máxime, si se trata de sociedades en vías o pretensiones de desarrollo.

Es evidente que el autor no considera aspectos relacionados con las consecuencias psicológicas y emocionales que este problema trae consigo, las mismas que estarían relacionadas con la instauración de un clima de perenne desconfianza mutua dentro de la sociedad que la padece. Es así, que en un ambiente donde la conducta corrupta se halla generalizada se podría generar un incremento de la violencia, sentimientos de indefensión y desesperanza, pues al percibir la situación de corrupción en la atmósfera social las personas bien podrían incrementar sus estados de estrés así como predisponer a brotes masivos de cuadros depresivos, porque tales son reacciones que se originan a causa de realidades que se perciben como insuperables y cuyos sentimientos de frustración por no poder cambiar el curso de un estado crítico (como la muerte de un ser querido, la pérdida de un lazo afectivo, o la imposibilidad de superar una plaga social como la corrupción), que se percibe y vive de modo constante. Hechos que ameritan mayor investigación para un conocimiento vasto y objetivo del mismo.

\section{ESTUDIOS PSICOLÓGICOS SOBRE LA CORRUPCIÓN EN EL PERÚ}

Son diversos los estudios que se han realizado con el fin de conocer en profundidad la naturaleza de este problema social. Es así, que existen investigaciones propias de la sociología, historia, ciencias políticas y economía.

La complejidad del fenómeno justifica el estudio realizado desde diversas disciplinas científicas, pues el problema de la corrupción tiene variantes sociales, económicas, políticas, etcétera, entre otras.

Así como las variantes mencionadas, no se puede soslayar la dimensión psicológica de la corrupción, pues el acto mismo comporta una conducta, y toda conducta incluye 
elementos cognitivos, afectivos, valorativos, actitudinales, etcétera, que son de tanta o mayor trascendencia que las de orden ético, económico, político, entre otras dimensiones que incluye este problema, porque se encuentran en la raíz o el origen mismo de su práctica social con los perjuicios implícitos a que predispone.

Sin embargo - aunque parezca extraño-, el estudio de la conducta corrupta no ha sido realizado dentro de la ciencia psicológica en la dimensión y necesidad que esta demanda (conociendo de sus efectos nocivos para la sociedad). Siendo tan solo unos cuantos los estudios psicológicos desarrollados sobre la corrupción en nuestro país: unos limitados respecto de la perspectiva teórica, sustentados en análisis bajo la teoría psicoanalítica y otros — con intención más objetiva - sobre la percepción de la corrupción en nuestro país, en base a estudios descriptivos. Estos estudios devienen insuficientes, tanto en la dimensión teórica y la metodológica, porque ninguno de los mismos permite explicar la génesis psicológica de la conducta corrupta, en razón de lo siguiente:

a) La corrupción desde el psicoanálisis:

Uno de los estudios más difundidos en nuestro medio es el realizado por el psicoanalista Saúl Peña K. (2007), quien en su artículo: «Reflexiones sobre la corrupción, patología mental, social, política y ética», refiere que la potencialidad que tenemos todos los seres humanos de ser corruptibles, «proviene del aspecto destructivo del instinto de muerte», que sería facilitado por «factores psicoplásticos (Sic.)», que tendría por finalidad infligir la muerte simbólica de alguien, «a través de la agresificación (Sic.) destructiva del instinto de vida» (Peña, 2007, p. 4). Asimismo, refiere que la «corrupción tiene que ver con nuestra historia vincular primigenia [...] con aspectos corruptógenos (Sic.) maternos, paternos, de la pareja, familiares, educativos, laborales, sociales, culturales, religiosos, políticos, económicos, ideológicos, etc.» (Peña, 2008).

Análisis como el mencionado, en su afán de explicar lo que hasta ahora resulta inexplicable para la ciencia psicológica, no hacen más que hundir el estudio psicológico de la conducta corrupta bajo un halo metafísico que 
resulta siendo inexplicable, pues encierra el problema bajo capas inextricables de conceptos sublimes y abstractos que, en lugar de aproximarnos, nos alejan del conocimiento del problema. En vista de que conceptos como "instinto de muerte", "factores psicoplásticos", “agresificación destructiva” (Peña, 2007, p. 3-4), carecen de definición científica y, per se, sus conceptos —lejos de explicar el problema-, lo obnubilan, oscureciéndolo innecesariamente. Recordemos que la explicación científica no solo se sustenta en la comprensión del problema sino en el uso claro y preciso de los conceptos; esto es, ausente de ambigüedades, de manera que la comunidad científica pueda entender y falsar sus hipótesis en pro de llegar a una verdad más certera de los hechos que son materia de explicación.

Siendo así, las explicaciones con fondo psicoanalítico como el mencionado, carecen de validez en sus procedimientos para explicar la conducta corrupta. No obstante, es importante destacar las aseveraciones en torno a la relación de la conducta corrupta con la experiencia recibida de quien la ejerce por parte del ambiente familiar, social, económico y cultural en el que se desarrolla. Sin duda, al parecer la relación puede asumirse como válida, sin embargo, la misma necesita mayor investigación, porque en el nivel de la aseveración tendrían el valor de hipótesis que necesitan ser falseadas en la experiencia, haciendo uso del método hipotético-deductivo propio de la ciencia y de la psicología en tanto disciplina científica.

b) Investigaciones cuantitativas en la ciudad de Lima - Perú:

Por otro lado, es necesario destacar los estudios realizados por el psicólogo Luis Vicuña Peri, Et. Al. (2007, 2008) sobre la corrupción en la ciudad de Lima.

Sus estudios «Disposiciones psicológicas hacia la corrupción en escolares y universitarios de la ciudad de Lima» (Vicuña, Et. Al. 2006), y «Percepción, tipos y medidas de control de la corrupción, según el sexo, ciclo académico y la facultad a la que pertenecen los estudiantes universitarios» (Vicuña, Et. Al. 2004), consisten en análisis descriptivos de las disposiciones hacia la 
corrupción y la percepción de la corrupción en escolares y universitarios de la ciudad de Lima.

Es de considerar, el esfuerzo realizado en la metodología procedimental de dichas investigaciones, que tratan de ceñirse a la objetividad de los hechos sobre datos cuantitativos - como las frecuencias relativas y análisis correlacionales_-; sin embargo, las conclusiones a las que se arriban, como:

- La importancia que cobran los actos corruptos son altamente significativas en la medida que aumenta la edad.

- Los púberes adolescentes y jóvenes atribuyen que los actos corruptos son susceptibles de ser manejados por ellos mismos

- La conceptualización de la corrupción es heterogénea, los estudiantes proporcionan hasta diez conceptos diferentes sin encontrar diferencias en función al sexo, a la facultad a la que pertenecen ni el ciclo académico (Vicuña, Et. Al. 2004 y 2006), entre otras.

Si bien tales conclusiones brindan información importante que podrían servir como base para futuras investigaciones, no explican la génesis psicológica de la conducta corrupta, más aún, considerando la metodología empleada, un estudio limitado a un recuento de respuestas en una encuesta, que es como pretender comprender la conducta adictiva sobre la base de sus percepciones en la población joven del Perú, las mismas que han de resultar negativas (pues es poco probable que los jóvenes acepten como sana y benéfica la adicción a las drogas, por ejemplo), pues sus consecuencias son, asimismo, negativas. De ahí que estudios de esta naturaleza tiendan a brindar información poco trascendente para comprender, y explicar, este problema social.

Por otra parte, es importante destacar que este estudio se desarrolló hace nueve años y en una población que no es representativa de la realidad nacional, 
sumando a las carencias del aporte teórico al estudio de la psicología de la corrupción, el hecho de estar desfasadas para nuestro tiempo y no representar a la mayoría de la población nacional; debiendo realizarse, por lo mismo, estudios actualizados, con datos pertinentes y en poblaciones descentralizadas (considerando que el problema de la corrupción es uno de tipo descentralizado que agobia a todo el Perú, desde la administración en la ciudad capital hasta la de los pueblos más alejados).

c) Balance de estudios

Estos y otros vacíos se encuentran en los estudios hasta hoy realizados sobre la psicología de la corrupción en nuestro país, los unos abundan de explicaciones metafísicas sustentadas en supuestos psicoanalíticos sin ningún rigor científico, y los otros, en datos preliminares que no alcanzan para explicar la problemática desde la psicología, y así brindar luces de solución para evitar su expansión patológica, tanto en nuestro país como en la sociedad en general.

Bajo la consigna de que lo que no representa una solución del problema, es parte del mismo, se puede aseverar, que los estudios psicológicos en esta línea de análisis se encuentran en una fase inicial debiéndose realizar investigaciones con mayor profundidad y rigor científico.

\section{ALTERNATIVAS METODOLÓGICAS PARA FUTURAS INVESTIGACIONES}

En tal sentido, apercibiendo los vacíos encontrados, se propone realizar investigaciones que consideren los siguientes puntos — dentro de su metodología y asunciones epistémicas—, de sus procedimientos del planteamiento del problema, hipótesis, recolección y análisis de resultados:

$1^{\circ}$ Siguiendo el método hipotético-deductivo de la psicología: como todas las ciencias fácticas (sociales y naturales) es necesario partir de postulados teóricos — dentro de la ciencia psicológica - con sustento empírico; esto es, teorías científicas de la psicología para la generación de hipótesis que deberán ser 
pasibles de ser falsadas en el proceso de la investigación, siguiendo el rigor y objetividad del método científico.

$2^{\circ}$ Tales postulados, dada la naturaleza de la problemática a evaluar y analizar, la misma que contiene, tanto elementos subjetivos y objetivos, se deberían fundamentar en la psicología cognitiva y el conductismo, respectivamente, pues los mismos no solo tienen validez científica en sus leyes y teorías, sino que además, aplican para el estudio de fenómenos relacionados con la conducta y la subjetividad asociada a ella.

$3^{\circ}$ Los estudios transversales — cuantitativos, cualitativos o mixtos-, si bien brindan información importante, son limitados y sesgados, pues se ciñen a un momento en particular dentro de la casuística y proceso del problema, lo que imposibilita conocer la 'génesis evolutiva' de las conductas corruptas y los factores asociados a ella, esto debido a que los estudios se realizan cuando el fenómeno ya ha ocurrido, ya se ha presentado; debiendo tan solo conocer las percepciones de los mismos, sin mayores pretensiones de identificación de su origen ni, mucho menos, de su explicación objetiva.

$4^{\circ}$ En tal sentido, se deberían realizar estudios longitudinales de naturaleza experimental, en niños, adolescentes y adultos, con el fin de tener un conocimiento más vasto y profundo de este comportamiento, tomando como ejemplo la metodología utilizada por el psicólogo suizo Jean Piaget, en sus estudios sobre el desarrollo cognitivo; cuya metodología marcó un paradigma en el estudio del comportamiento humano considerando variables cognitivas en su curso de desarrollo, el mismo que consistió en diseños de investigación en series de análisis longitudinales.

$5^{\circ}$ En vista de que la explicación científica lleva consigo la posibilidad de la predicción per se, realizar estudios psicológicos que busquen explicar la naturaleza y génesis de la conducta corrupta llevaría consigo la posibilidad de estar en condiciones de predecir su ocurrencia en una $u$ otra persona, de manera que se podrían evitar, y con ello prevenir, la ocupación de cargos relevantes de la administración pública a individuos con una alta tendencia hacia los actos de 
corrupción, evitando así la pérdida de ingentes cantidades de recursos económicos y materiales en los países más afectados por dicho problema.

$6^{\circ} \mathrm{Al}$ ser la corrupción, un problema multicausal, se deberían planificar estudios interdisciplinarios; esto es, desarrollar investigaciones con la participación mancomunada de otras ciencias — sociales y naturales_-, como la sociología, neuropsicología, etología, antropología, economía, criminalística, etc. De manera que se tenga un panorama mucho más vasto de posibilidades explicativas y, per se, predictivas.

\section{d) CONCLUSIONES Y RECOMENDACIONES}

Habiendo realizado el análisis sobre la temática que convoca el presente ensayo, se pueden expresar las siguientes conclusiones:

a. Es necesario y de suma importancia realizar estudios psicológicos de la conducta corrupta en nuestro país, al tratarse de un problema de ingentes consecuencias nocivas para su desarrollo social, político, económico y material.

b. Los estudios psicológicos realizados sobre la corrupción, son todavía de naturaleza subjetiva y superficial, estando ausentes las investigaciones ceñidas al método científico que se orienten a la explicación del problema, no tan solo a su especulación subjetiva ni a la percepción social de su naturaleza y ocurrencia.

c. Los estudios que se realicen deben estar sustentados en teorías científicas de la psicología, sobre las que deben fundamentarse las hipótesis a ser 'falsadas', tomando como base al método hipotético-deductivo, propio de las ciencias fácticas, en la que se encuentra inmersa la psicología, en tanto ciencia social con sustento teórico-científico y empírico.

d. La metodología prioritaria debe ser la experimental, en series de estudios longitudinales, no solo transversales, pues la primera permite conocer, además de la naturaleza del problema, también su 'origen y proceso' evolutivo, conjuntamente con los factores asociados a ellos. 
e. Así como se deben realizar estudios psicológicos con sustento exclusivo de teorías científicas propias de la psicología, al hacer evocación de la naturaleza sistemática del conocimiento científico, se deben trazar investigaciones interdisciplinarias en asociaciones teórico-metodológicas con otras ciencias sociales y naturales, pues mediante las mismas, se pueden dar mayores luces sobre la naturaleza de este fenómeno al tratarse de un problema complejo dada su multicausalidad.

f. Es pertinente hacer hincapié en la necesidad de la generalización de los estudios que se realicen en la psicología de la corrupción, pues la ciencia se sustenta en información sobre datos constantes a manera de leyes y teorías científicas que le permitan, la explicación y predicción de la conducta humana para la comprensión, en este caso singular, de la conducta corrupta y de la capacidad de predicción de su ocurrencia, de manera que se pueda evitar y, con ello, impedir sus efectos nocivos para la sociedad y el desarrollo político, económico, social, de salud y bienestar de nuestro país.

g. En vista de los estudios desarrollados, se ha puesto en evidencia que el comportamiento corrupto es evaluado cada vez más como un 'fenómeno normal', es importante conocer la magnitud de esta percepción en poblaciones de niños, adolescentes, jóvenes y adultos; de manera que se tenga una visión más general sobre este hecho, que - de confirmarse su presencia en las poblaciones mencionadas-, señalarían una tendencia bastante negativa que no haría más que agravar el problema, pues frente a un hecho que se apercibe patológico, no existe peor contexto que aquel que lo acepta como normal o normativo, que sería semejante a concebir como un hecho normal el robo o la estafa.

h. Asimismo, es importante realizar investigaciones psicológicas sobre las repercusiones de la conducta corrupta y su percepción social en la salud emocional de la sociedad, pues si este problema social se halla generalizado en todas las esferas de la administración pública, podrían generar en la sociedad una atmósfera de inseguridad y desconfianza perenne en la población general, predisponiendo a un incremento en las tasas de estrés, ansiedad y 
desesperanza frente a la propia percepción de incapacidad de cambiar o revertir el problema; dando lugar, asimismo, a ser parte del problema, en la posibilidad de obtener ganancias; esto es, caer en el pensamiento de que: «si todos son corruptos y obtienen ganancias, qué caso tiene oponerse si todo el sistema está corrompido y si siendo parte del problema se pueden obtener beneficios propios». Un hecho a todas luces preocupante y alarmante, que amerita mayor investigación.

i. Por todas las razones mencionadas, tanto en la discusión y el análisis del presente estudio, se concluye - a manera de síntesis-, que la investigación de la corrupción dentro del espacio científico de la psicología, necesita mayor desarrollo, tanto en la cantidad de los estudios, como en la elección de metodologías objetivas y con el debido rigor epistémico bajo la consigna de lograr su explicación y con ello, dar luces para mitigar su aparición y, per se, decrementar sus efectos tan nocivos y perjudiciales en la sociedad peruana, en particular, y la sociedad global, en general.

\section{BIBLIOGRAFÍA}

Basadre, J. (2005). Historia de la República del Perú (1822 - 1933). Lima, Perú: El Comercio.

Contraloría General de la República. (2014). Premier dio cuenta de pérdidas por la corrupción. Recuperado de:

https://apps.contraloria.gob.pe/conferenciaanticorrupcionperu/2014/medios.h tml

González, A. (1985). Dimensiones de la corrupción. Revista de psicología, volumen (3), 23-40.

Instituto de Democracia y Derechos Humanos (2012). Estadísticas sobre la corrupción. Recuperado de:

http://idehpucp.pucp.edu.pe/anticorrupcion/estadisticas-sobre-la-corrupcion/

Macera, D. (5 de julio del 2105). ¿Cuál es el costo de la corrupción en el Perú? El Comercio. Recuperado de http://elcomercio.pe/politica/actualidad/cual-costocorrupcion-peru-informe-noticia-1820300

Nizama, M. (2008). La corrupción: psicopatología. Revista de Investigación en Psicología, volumen (11), 201-213. http://sisbib.unmsm.edu.pe/bvrevistas/investigacion_psicologia/v11_n1/pdf/ a02.pdf 
Peña, S. (2007). Reflexiones sobre la corrupción, patología mental, social, política y ética. Revista de Investigación en Psicología, volumen (10), 163-171. http://sisbib.unmsm.edu.pe/bvrevistas/investigacion_psicologia/v10_n1/pdf/ a10.pdf

Real Academia de la Lengua Española. (2014). Diccionario de la lengua española. Recuperado de: http://www.rae.es/recursos/diccionarios/drae

Transparency International. (2013). Global Corruption Barometer. Recuperado de: http://issuu.com/transparencyinternational/docs/2013_globalcorruptionbaro meter_en?e=2496456/3903358\#search

United Nations Office on Drugs and Crime. (2015). UNODC and corruption. Recuperado de http://www.unodc.org/lpo-brazil/en/corrupcao/index.html

Vicuña, L. Et. Al. (2004). Disposiciones psicológicas hacia la corrupción en escolares y universitarios de la ciudad de Lima. Revista de Investigación en Psicología, volumen (7), 1-25. http://dx.doi.org/10.15381/rinvp.v7i1.5143

Vicuña, L. Et. Al. (2006). Percepción, tipos y medidas de control de la corrupción, según el sexo, ciclo académico y la facultad a la que pertenecen los estudiantes universitarios. Revista de Investigación en Psicología, volumen (9), 1-28. http://sisbib.unmsm.edu.pe/bvrevistas/investigacion_psicologia/v09_n2/pdf/ a04v9n2.pdf 